Revista Signos

2010, 43(72)

$125-152$

\title{
Elecciones linguiísticas en el proceso de planificación de un ensayo
}

\author{
María Nélida Pérez \\ Universidad Simón Bolívar \\ Venezuela
}

Resumen: En el presente artículo se reporta una investigación que tuvo como objetivo determinar los niveles de realización y factores de motivación de las elecciones lingüísticas en la escritura de un esquema de ideas, siguiendo un formato de planificación de un ensayo breve. La muestra estuvo constituida por doce estudiantes de primer año de la Universidad Simón Bolívar, Venezuela. El corpus objeto de estudio estuvo formado por los esquemas de los estudiantes, además de los ensayos, como recursos de apoyo que permitieron evaluar la propiedad de intertextualidad entre texto y ensayo (presencia/ausencia de cada argumento en el ensayo y fuerza del argumento en el ensayo). Los resultados dan cuenta de un claro manejo intencional (estratégico) en la toma de decisiones lingüísticas opcionales en todos los niveles de realización del enunciado oracional y de la interrelación de factores de orden pragmático, textual y cognitivo.

Palabras Clave: Escritura, argumentación, elecciones lingüísticas.

Recibido: 16-VI-2008

Aceptado: 24-VIII-2009
Correspondencia: María Nélida Pérez (mnperez@usb.ve). Departamento de Lengua y Literatura, Universidad Simón Bolívar. Edificio de Estudios Generales, piso $3^{\circ}$, Valle de Sertenejas, Baruta, CP 89000, Eduardo Miranda, Venezuela. 


\title{
Linguistic choices in the planning process of an essay
}

\begin{abstract}
This article reports research whose aim is determine levels of accomplishment and motivating factors of the linguistic choices in the writing of a plan of ideas, as the planning format for a brief essay. The sample was obtained from twelve Universidad Simón Bolívar, Venezuela, freshmen students. The corpus was made up of the schemes written by the students, as well as the essays as support resources that helped evaluate the intertextuality between text and essay (presence/ absence of each argument in the essay and the strength of each argument in the essay). The results show a clear intentional (strategic) mastery in both the optional linguistic decision-making at all the instantiation levels of the sentence statement and the relationship of the pragmatic, textual and cognitive levels.
\end{abstract}

Key Words: Writing, argument, linguistic choices.

\section{INTRODUCCIÓN}

Las investigaciones en escritura, en cualquiera de los niveles del sistema educativo, han tendido a examinar las habilidades de los estudiantes en aspectos relacionados básicamente con decisiones lingüísticas regidas por criterios de gramaticalidad y aceptabilidad, ya sea referentes a la macroestructura -como la elaboración de resúmenes (Arnoux, Nogueira \& Silvestri, 2006; Beke \& Bruno, 2005; Serrano, 2003), textos argumentativos (Poblete, 2005; Villegas, 2001) o expositivos (Fuenmayor, Flores \& Rincón, 2008)- o a la microestructura textual, que requiere de diversas decisiones gramaticales (Morles, Arteaga, Bustamante \& García, 2002).

Los estudios realizados en los últimos años, dirigidos al análisis de la escritura académica, se mantienen en la línea del 'deber ser', esto es, de las decisiones que debe tomar el estudiante universitario para construir textos acordes con las exigencias de la comunidad de la que forma parte y en la que construye su saber. En el entendido de que la lectura y la escritura son procesos en los que se está en constante aprendizaje, de que los modos de leer y escribir difieren en los diferentes niveles y ámbitos educativos en los que se lee y escribe, y de que, por tanto, es necesario apoyar institucionalmente su enseñanza en todos los casos, surge esta nueva corriente de estudio. En este sentido, los trabajos de Carlino (2003, 2004), por ejemplo, definen la responsabilidad que tienen las universidades, y, dentro de ellas, cada disciplina académica, en la enseñanza de los modos de elaboración y comunicación del conocimiento a través de la escritura, a los estudiantes que ingresan ya sea para seguir estudios de grado o de postgrado (véase, además, Tapia, Burdiles \& Arancibia, 2003; Arnoux et al., 2006; Arnoux, Borsinger, Carlino, Di Stefano, Pereira \& Silvestri, 2004). Asimismo, Castelló (2007) recoge un conjunto de propuestas precisas y detalladas de varios autores, relativas tanto a las etapas de creación 
del texto académico, como a sus funciones y tipos e, incluso, a los factores emocionales que forman parte de esa tarea. De manera que el estudio de los procesos de escritura se enriquece a partir de nuevas visiones que privilegian su función epistémica y redimen al estudiante de cargar con la culpa de todas las deficiencias.

En esta investigación, desde una perspectiva integrada por enfoques pragmáticos, discursivos y cognitivos, iniciamos una indagación sobre la escritura académica, pero, específicamente, sobre las elecciones lingüísticas concebidas como producto: (a) de una propiedad del uso del lenguaje que es la variabilidad, esa potestad que tiene cada individuo de elegir dentro de un conjunto de posibilidades; y (b) de su conciencia reflexiva, pues tales elecciones son "hechas entre una serie de opciones, y por eso con un estatus conceptual o comunicativo específico" (Verschueren, 2002: 298).

\section{Uso del lenguaje: Una continua elección}

Desde la perspectiva pragmática desarrollada por Verschueren (2002: 110), “el uso del lenguaje debe consistir en la continua elección lingüística, consciente o inconsciente ${ }^{1}$, por razones internas (p. e. estructurales) y/o externas al lenguaje"; elecciones que se ubican en cualquiera de los niveles de la lengua (fonético/fonológico, morfológico, sintáctico, léxico o semántico), y que suponen la consideración de variaciones de tipo regional, social o funcional. De modo que este proceso también implica seleccionar estrategias comunicativas, lo que incide, a su vez, sobre la elección del estilo y de las formas lingüísticas del enunciado. Así, pues, las elecciones no son equivalentes; existen opciones preferentes y no preferentes en función de los acuerdos establecidos socialmente. Tan compleja realidad lingüística lleva a afirmar a Verschueren (2002) que existe la necesidad de elaborar una teoría del uso del lenguaje que pueda explicar tales elecciones. Para ello, propone la consideración de tres conceptos clave interrelacionados: variabilidad, negociabilidad y adaptabilidad.

La variabilidad "es la propiedad del lenguaje que define la gama de posibilidades dentro de las cuales se puede seleccionar" (Verschueren, 2002: 115); tal conjunto de posibilidades está en permanente cambio y no solo debido a la dimensión diacrónica de la lengua. En cualquiera de los momentos de la enunciación, del intercambio, los sujetos pueden desechar alternativas y optar por otras, según lo requieran para hacer efectivas sus intenciones comunicativas. Gracias a la propiedad de negociabilidad, no eligen "mecánicamente o según unas reglas estrictas o unas relaciones de forma-función fijas, sino basadas en principios altamente flexibles" (Verschueren, 2002: 116), prácticos, reales (en contraposición a 'posibles'). Ahora bien, en una situación comunicativa, deben elegir, aunque no dispongan de las mejores posibilidades, entre 
otras razones, porque se ven restringidos a operar con opciones convencionales o habituales; de allí que algunas formas puedan adquirir nuevos usos con la finalidad de satisfacer nuevas necesidades. Es la propiedad de adaptabilidad la que:

“capacita a los seres humanos a hacer elecciones lingüísticas dentro de una gama de posibilidades variable de modo que se acerquen a la satisfacción de sus necesidades comunicativas" (Verschueren, 2002: 119).

Evidentemente, lengua, código y estilo son los tres niveles superiores de elección lingüística. En correspondencia con las definiciones de lengua y código ${ }^{2}$, en tanto niveles estructurales que muestran algún tipo de variabilidad en el uso de la lengua, y por ende, objetos de elección lingüística. Verschueren (2002: 201) establece una noción de estilo:

"Usaremos estilo para describir la variabilidad a lo largo de las dimensiones de 'formalidad e informalidad', del habla informal o coloquial a usos altamente formales. Todas las lenguas son susceptibles de usos estilísticos variables y también la mayoría de códigos, aunque algunos de ellos (como argots o jergas) se encuentran normalmente en el extremo de informalidad, mientras otros (como el legal) son típicamente formales".

Desde la perspectiva del análisis del discurso, van Dijk (2000: 35) plantea otra noción que bien puede relacionarse con la anterior:

“Para describir los mismos sucesos pueden también [esto es, además de variaciones léxicas] utilizarse variaciones en la pronunciación, en la escritura, en los elementos visuales o en los gestos que acompañan al discurso, en el orden de las palabras, en el orden de las oraciones: todas estas variaciones funcionales forman parte del estilo. En síntesis, el estilo es habitualmente una variación dependiente del contexto del nivel de expresión del discurso".

Ambas definiciones nos permiten fundamentar teóricamente la descripción de las elecciones estilísticas (en el sentido de van Dijk, 2000) obradas por los sujetos de la muestra; sujetos diferentes, al tiempo que estudiantes de un mismo curso universitario, en la escritura de un mismo tipo de texto (esquema de ideas) que posee una misma superestructura (tesis y argumentos) y que forma parte de la misma operación del proceso de escritura (planificación de un texto argumentativo, específicamente, de un ensayo), a partir de un mismo tópico (resistencia del libro en formato impreso ante el embate de la escritura digital), en un mismo contexto (el académico y, por tanto,) de formalidad discursiva (estilística, en el sentido de Verschueren, 2002). En consecuencia, dentro de este estilo, el individuo podrá optar (a) en el nivel macroestructural, por apoyar una posición u otra, por desarrollar su posición con argumentos propios o compartidos (ya sea parcial o totalmente) con sus compañeros, todos relacionados con un 
mismo tópico; y (b) en el nivel microestructural, por el uso de una construcción en lugar de otra o por un término en lugar de otro.

Asumimos, como se hace evidente, el ampliamente conocido modelo de comprensión de Kintsch y van Dijk (1978) en el que la estructura semántica se compone de un nivel macroestructural, donde reside el contenido general del discurso, el tema o asunto del que trata, y el microestructural, que comprende el nivel local del discurso, esto es, la estructura de las oraciones individuales y de sus relaciones.

De acuerdo con Verschueren (2002), la organización progresiva del discurso exige establecer dos vertientes en cuanto a las selecciones que el individuo hace al establecer las relaciones entre los constituyentes del enunciado; la primera está referida a los niveles de organización estructural y, la segunda, a la construcción de los enunciados y, por lo tanto, tiene que ver con los principios que los rigen. Verschueren (2002) recoge los ya conocidos niveles de elección lingüística, que -como hemos dicho- van desde los más generales, lengua, código y estilos, hasta los referidos propiamente a la construcción del enunciado: sonidos, morfemas y palabras, cláusulas, oraciones y proposiciones. En lo concerniente a los principios que rigen la construcción de enunciados, distingue dos tipos según su nivel: enunciados oracionales y enunciados supraoracionales. En ambos casos, considera la forma en que el significado se presenta y se desarrolla, aunque le atribuye un papel más activo, como organizador discursivo en los enunciados supraoracionales debido a la relación que establece entre niveles de organización del discurso y capacidad de procesamiento por parte del sujeto.

\section{Escritura y actividad metalinguística}

Los diferentes modelos y enfoques didácticos socio-cognitivos que en torno a la escritura han surgido durante los últimos años la han definido como un proceso, y han centrado su atención en describir cómo este proceso se lleva a cabo, qué operaciones entran en funcionamiento, cómo se interrelacionan y cuáles son las estrategias que el escritor sigue a lo largo de su elaboración discursiva; esto, evidentemente, ha significado importantes aportes tanto en el campo de teoría de la escritura como de su aplicación didáctica. Dos modelos que han comandado esta visión son los ampliamente conocidos de Hayes y Flower (1980), Flower y Hayes (1996) y Scardamalia y Bereiter (1992). Los primeros conciben la escritura como un proceso recursivo donde la operaciones básicas de planificación, textualización y revisión, verificadas mediante un mecanismo de control, no se presentan en forma de etapas porque, a medida que escriben, los escritores continuamente 'pre-escriben y re-escriben'. Desde ambos enfoques, la escritura se convierte en un espacio para la solución de problemas; el escritor debe iniciar su texto con el 
planteamiento de un problema cuya solución le permitirá lo que Scardamalia y Bereiter (1992) han denominado la 'transformación de su conocimiento' y, por ende, el aprendizaje al que se llega a través del poder epistémico de la escritura.

El disponer de estrategias que permitan 'hacer' elecciones discursivas de diferente índole es fundamental en la escritura académica y, particularmente, en la escritura de textos argumentativos, debido, entre otras razones, a la intención que mueve a sus emisores, esto es, persuadir o convencer. Como expresa van Dijk (2000: 36), “la 'función’ persuasiva del texto (...) puede depender del estilo, del sentido o de la coherencia”, de modo que el individuo tiene que atender a varios aspectos y evaluar la selección de las proposiciones por incorporar al tiempo que la estructura textual de cada enunciado oracional, en función de un estilo formal que exige, en mayor medida que el informal, la puesta en práctica de mecanismos de representación y control (Bialystok, 2001). Esto hace que active o incremente de manera considerable su actividad metalingüística ${ }^{3}$ o, lo que es lo mismo, su "actividad discursiva sobre el lenguaje", siguiendo la definición dada por Camps, Guash, Milian \& Ribas (2000: 137), quienes parten de los modelos de desarrollo cognitivo propuestos por Karmiloff-Smith (1994) y Gombert (1992).

Karmiloff-Smith (1994) concibe la existencia de actividad metalingüística a partir del momento en que la información presente 'en' la mente se transforma en conocimiento 'para' la mente, a través de varias fases de redescripción. El conocimiento explícito al que se tiene acceso de manera consciente, en el último de los niveles, se rerrepresenta a través de un código muy cercano al lenguaje natural, con la finalidad de que pueda ser traducido con facilidad a un formato verbalmente expresable. Gombert (1992), quien elabora su modelo a partir del de Karmiloff-Smith (1994), define la actividad metalingüística como 'cognición acerca del lenguaje'; cognición marcada por un doble carácter, reflexivo e intencional, pues se reflexiona sobre el lenguaje y su uso con la finalidad de planificar y controlar su uso.

De acuerdo con Gombert (1992), tal actividad puede manifestarse en cualquiera de los niveles de la lengua: 'metasemántica', al manipular palabras o sintagmas sin alterar el significado; 'metatextual', al manejar y monitorear el procesamiento del texto, lo que incluye la coherencia, la cohesión y la estructura global; y 'metapragmática', al reflexionar sobre -y, por tanto, monitorear- los parámetros extralingüísticos, situacionales, en los que el enunciado se realiza y que inciden directamente sobre su producción y comprensión.

A partir de este nuevo acercamiento al desarrollo del conocimiento, muchas investigaciones en el ámbito de la escritura (Camps \& Ribas, 1996; Camps, 2001; Castelló \& Monereo, 1996; Milian, 1996; Milian \& Camps, 2000; Miras, 2000; Riestra, 1999; Velásquez \& Alonzo, 2007) han tendido a crear nuevas propuestas didácticas que tienen como objetivo promover la consideración de 
este proceso como un hecho comunicativo, lo que Allal (2000) ha llamado 'cognición situada' o 'saber metacognitivo en situación', basado en un modelo de aprendizaje orientado a promover la adquisición de mecanismos de autorregulación en relación con el contexto comunicativo -real o simulado 'del modo más convincente posible'- en el que las tareas de escritura se producen. Son muchos ya los estudios que en las dos últimas décadas se han orientado en esta línea en que la lengua cumple una función comunicativa, como instrumento de uso y al mismo tiempo reflexiva, como instrumento y objeto de aprendizaje.

\section{Metodología}

\subsection{Objetivo y problemas}

Nuestro propósito, en esta investigación, fue determinar los factores de incidencia y los niveles de realización de las elecciones lingüísticas por las que optó una muestra de estudiantes universitarios con la finalidad de autorregular sus procesos de escritura, durante la elaboración, en grupo, de un esquema, formato de planificación de un ensayo. En consecuencia, nos planteamos dos problemas específicos de estudio: (a) ¿En qué niveles de realización del texto operan las elecciones lingüísticas, entendidas como producto de la actividad metalingüística desplegada por el sujeto?; y (b) ¿Qué factores pueden motivar la elección de una opción sobre otra en los diferentes niveles?

\subsection{Los Sujetos}

Los sujetos fueron doce estudiantes de Ingeniería (de diferentes especialidades), del segundo trimestre de primer año, de la Universidad Simón Bolívar de Venezuela, cursantes de la asignatura Lenguaje II, y con una edad promedio de 17.6 años. Los estudiantes, al ingresar, deben cursar un Ciclo Básico de un año de duración, con materias coincidentes para todas las carreras de Ingeniería; posteriormente se incorporan al Ciclo Profesional, con materias ya diferenciadas por carrera. El régimen de estudios es trimestral y en cada trimestre del Ciclo Básico deben cursar un Lenguaje y una Matemática, además de otras asignaturas como Física, Idiomas y Ciencias Sociales.

\subsection{Procedimiento}

Esta actividad formó parte de una secuencia didáctica cuyo objetivo fue desarrollar competencias discursivas para la escritura de un ensayo. La secuencia constó de varias fases que incluyeron la escritura de un esquema (planificación) y de un ensayo a partir de tal esquema, 
la lectura de textos inherentes al tema y la reescritura de ambos textos, sin embargo, aquí nos centraremos en las referidas a una parte de la fase inicial: planificación del ensayo a partir de un esquema de ideas; por tanto, las elecciones que se describirán serán tomadas del esquema. Esta operación de planificación tiene como fin último la planificación del texto por escribir, a través de una finalidad primera, la elaboración del esquema de tal texto, proceso que encierra, a su vez, las tres operaciones implicadas en el acto de escribir (planificación, escritura y revisión). De manera que se establecen dos niveles de actuación: planificar la escritura de un texto $B$ planificando, escribiendo y re-escribiendo un texto $A$.

En cuanto al procedimiento utilizado, en una primera clase planteamos a los estudiantes una pregunta tomada del discurso Eco con motivo de la inauguración de la Biblioteca de Alejandría (2003): ¿Resistirán los libros el embate de la escritura digital? Asimismo, les indicamos que debían buscar información que les permitiera justificar la posición crítica que, frente a ese problema, asumirían en la escritura de sus ensayos. En la clase siguiente, los estudiantes se reunieron en grupos (tríos) con la finalidad de crear un esquema de escritura estructurado en tesis y argumentos. El partir de una misma pregunta (tópico) así como generar una discusión entre los estudiantes durante la elaboración de sus esquemas, de alguna manera, nos permitió asegurar la construcción de un mismo discurso sobre el que cada sujeto podía obrar variaciones estilísticas. En palabras de van Dijk (2000: 35):

"Los significados son los mismos: de lo contrario no hablaríamos de una variante estilística del discurso, sino de un discurso diferente. Pero aun así, existen variaciones estilísticas: se puede hablar de los mismos tópicos o sucesos (referentes) y hacerlo con distintos sentidos locales (detalles) (...). En otras palabras, el concepto de estilo supone usualmente que al menos algo (el sentido, el tópico, los sucesos) permanece igual, de modo que podamos comparar 'cómo' los discursos dicen lo mismo".

Una vez concluida la discusión y elaborado el esquema ( 2 horas de clase), fue recogido. En la sesión siguiente, los entregamos y, recordando el carácter flexible que todo esquema tiene, les pedimos su revisión en atención a los siguientes parámetros: claridad en la exposición de la tesis y de los argumentos, organización coherente de los argumentos, coherencia entre la tesis asumida y los argumentos seleccionados para desarrollarla y aspectos gramaticales. Finalmente, les solicitamos la redacción del ensayo en un tiempo máximo de dos horas.

\subsection{Corpus}

El corpus de la investigación está formado por los esquemas de doce estudiantes. Contamos, además, con los ensayos como recursos de apoyo, con la finalidad de analizar la relación entre 
los argumentos del esquema y su tratamiento en el ensayo: presencia o ausencia de cada argumento en el ensayo (evaluación de la intertextualidad entre esquema y ensayo) y la fuerza de cada argumento en función del número de palabras utilizadas para desarrollarlo ${ }^{4}$, considerando el total de escritos relativos únicamente a la justificación del conjunto de argumentos, lo que nos da cuenta de la actividad metalingüística desplegada por el sujeto en la operación de planificación textual y de cómo operan los mecanismos de control al incluir consciente o inconscientemente cada argumento en el ensayo.

Para efectos de su identificación en la presentación y análisis del corpus, los estudiantes fueron designados con la letra $\mathrm{E}$ y dos números, el primero en función del grupo al que pertenecían y el segundo, de su posición en el grupo. Así, por ejemplo, E1.1 es el primer estudiante del grupo 1 y E3.2, el segundo del grupo 3. Asimismo, fueron utilizadas las iniciales ( $T$ ) y $(A)$ para indicar la tesis y los argumentos, respectivamente.

\section{Presentación y análisis de resultados}

Los datos generales presentes en las Tablas 1 y 2 dan cuenta de los resultados obtenidos en relación con: (a) el sentido de la proposición o, más específicamente, de la macroproposición (MP), por cuanto el esquema representa la estructura semántica global del texto por escribir, y (b) la forma del enunciado oracional (EO), tanto en la tesis como en los argumentos. En ambas tablas, se registra la coincidencia, total (CT) o parcial (CP), o la no coincidencia (NC). 
Tabla 1. Tipo de coincidencia, total (CT), parcial (CP) o no coincidencia (NC), en lo relativo a la macroproposición (MP) y a la forma del enunciado oracional (EO) de la tesis.

\begin{tabular}{|c|c|c|}
\cline { 2 - 3 } \multicolumn{1}{c|}{} & \multicolumn{2}{c|}{ TESIS } \\
\hline Estudiante & MP & EO \\
\hline E1.1 & CT & CP \\
\hline E1.2 & CT & CP \\
\hline E1.3 & CT & CP \\
\hline E2.1 & CT & CT \\
\hline E2.2 & CP & CP \\
\hline E2.3 & CT & CT \\
\hline E3.1 & CT & CT \\
\hline E3.2 & CT & CT \\
\hline E3.3 & CT & CT \\
\hline E4.1 & CP & CP \\
\hline E4.2 & CP & CP \\
\hline E4.3 & NC & NC \\
\hline
\end{tabular}

A primera vista, se perciben tres categorías: (a) coincidencia total en la macroproposición, pero parcial en la forma que presenta el enunciado oracional; (b) coincidencia total del grupo o de individuos de un mismo grupo y divergencia de otros tanto en la macroproposición como en la forma del enunciado oracional y; (c) coincidencia parcial entre las macroproposiciones y las formas de los enunciados oracionales entre algunos de los miembros del grupo o no coincidencia. Hemos optado, en cada categoría, por presentar los resultados de la investigación por grupo de estudiantes, lo que nos permite establecer los factores que motivaron sus elecciones lingüísticas a partir de la consideración de una misma información. Si bien puede haber fenómenos que se presenten en forma recurrente, en cada uno analizaremos los más resaltantes; esto nos da la posibilidad de explicarlos en detalle y, finalmente, concluir al respecto. 
Tabla 2. Tipo de coincidencia, total (CT), parcial (CP) o no coincidencia (NC), en lo relativo a las macroproposiciones (MC) y la forma de los enunciados oracionales (EO) de los argumentos.

\begin{tabular}{|c|c|c|c|c|c|c|c|c|c|c|c|c|}
\hline \multirow[b]{3}{*}{ Estudiante } & \multicolumn{12}{|c|}{ ARGUMENTOS } \\
\hline & \multicolumn{2}{|c|}{$\mathrm{A} 1$} & \multicolumn{2}{|c|}{ A2 } & \multicolumn{2}{|c|}{ A3 } & \multicolumn{2}{|c|}{ A4 } & \multicolumn{2}{|c|}{ A5 } & \multicolumn{2}{|c|}{ A6 } \\
\hline & MP & EO & MP & EO & MP & EO & MP & EO & MP & EO & MP & EO \\
\hline E1.1 & $\mathrm{CT}$ & CT & CT & $\mathrm{CT}$ & CT & CT & & & & & & \\
\hline $\mathrm{E} 1.2$ & $\mathrm{CT}$ & CT & CT & $\mathrm{CT}$ & CT & CT & & & NC & & & \\
\hline E1.3 & $\mathrm{CT}$ & CT & CT & $\mathrm{CT}$ & CT & CT & NC & & & & & \\
\hline E2.1 & $\mathrm{CT}$ & CT & CT & $\mathrm{CT}$ & $\mathrm{CP}$ & $\mathrm{CP}$ & & & & & & \\
\hline E2.2 & $\mathrm{CP}$ & NC & & & $\mathrm{CP}$ & NC & & & & & & \\
\hline E2.3 & $\mathrm{CT}$ & CT & CT & $\mathrm{CT}$ & $\mathrm{CP}$ & $\mathrm{CP}$ & & & & & & \\
\hline E3.1 & $\mathrm{CP}$ & $\mathrm{CP}$ & CT & $\mathrm{CP}$ & $\mathrm{CP}$ & $\mathrm{CP}$ & CT & $\mathrm{CP}$ & $\mathrm{CT}$ & $\mathrm{CP}$ & $\mathrm{CT}$ & $\mathrm{CP}$ \\
\hline E3.2 & $\mathrm{CT}$ & CT & CT & $\mathrm{CT}$ & CT & CT & CT & $\mathrm{CT}$ & $\mathrm{CT}$ & $\mathrm{CP}$ & CT & $\mathrm{CT}$ \\
\hline E3.3 & $\mathrm{CT}$ & CT & CT & $\mathrm{CT}$ & CT & CT & $\mathrm{CT}$ & $\mathrm{CT}$ & $\mathrm{CT}$ & $\mathrm{CP}$ & $\mathrm{CT}$ & $\mathrm{CT}$ \\
\hline E4.1 & $\mathrm{CT}$ & $\mathrm{CP}$ & $\mathrm{CP}$ & $\mathrm{CP}$ & CT & $\mathrm{CP}$ & $\mathrm{CP}$ & $\mathrm{CP}$ & $\mathrm{CP}$ & NC & & \\
\hline E4.2 & $\mathrm{CT}$ & $\mathrm{CP}$ & $\mathrm{CP}$ & $\mathrm{CP}$ & CT & $\mathrm{CP}$ & $\mathrm{CP}$ & $\mathrm{CP}$ & & & NC & NC \\
\hline E4.3 & NC & NC & NC & NC & & & & & $\mathrm{CP}$ & NC & & \\
\hline
\end{tabular}

\subsection{Coincidencia total en la macroproposición, pero parcial en la forma que presenta el enunciado oracional: Grupo 1}

En el grupo 1, los tres estudiantes coincidieron en estructurar la tesis ('el fuerte embate tecnológico modificará pero no eliminará la utilización del libro') en términos contraargumentativos: si bien es cierto que... también lo es que...; lo que muestra que parten, principalmente, de una realidad que creen incuestionable, "Internet facilita el proceso de localización de información", pero contra la cual argumentarán considerando su lado débil: "gran parte del material contenido en la red se encuentra sustentado por un formato físico".

Si bien es cierto que Internet facilita el proceso de localización de información de manera resumida y concisa, también lo es que la mayor parte del material contenido en ella, se encuentra sustentado por un formato físico, lo cual indica que el fuerte embate tecnológico modificará pero no eliminará la utilización del libro.

(E.1.2) Si bien es cierto que Internet facilita el proceso de localización de la información, por ofrecerla de manera resumida y concisa, también lo es que gran parte del material contenido en ella se encuentra sustentado por un 
formato físico, lo cual indica que el fuerte embate tecnológico modificará mas no eliminará la utilización del libro.

Si bien es cierto que Internet facilita el proceso de localización de la información por ofrecerla de manera resumida y concisa, también lo es que gran parte del material contenido en la red se encuentra sustentado por un formato físico, lo cual indica que el fuerte embate tecnológico modificará pero no eliminará la utilización del libro.

Las tres tesis concuerdan en tema y divergen en cuatro aspectos. El primero tiene que ver con las relaciones de coherencia: E1.2 y E1.3 coinciden en establecer una relación causa-efecto; E.1.1, en cambio, le proporciona otra función: la de atributo.

(E.1.2) $(\mathrm{E} 1.3)(\mathrm{T}) \quad$...Internet facilita el proceso de localización de la información, por ofrecerla de manera resumida y concisa...

(E.1.1) .... Internet facilita el proceso de localización de información de manera resumida y concisa...

El segundo está referido a aspectos lexicales. E1.1, a diferencia de E.1.2 y E1.3, prefiere la expresión “la mayor parte”, en lugar de "gran parte”; evidentemente, una 'gran parte' no necesariamente es 'la mayor':

(E.1.1) $\quad(\mathrm{T}) \quad$...la mayor parte del material...

(E.1.2) (E1.3) ...gran parte del material...

El tercero tiene que ver con relaciones de cohesión. Mientras E1.1 y E.1.2 utilizan una proforma, "ella", como estrategia de sustitución lexical de "Internet", E1.3 prefiere el empleo de un sustantivo, "red”, esto es, de una expresión definida:

$(E .1 .1)(E .1 .2) \quad(T) \quad$...material contenido en ella... ...material contenido en la red...

Finalmente, hay una transformación de tipo pragmático, estilístico, en el uso que hace E1.2 de "mas" en lugar de "pero", como un intento de formalización (refinamiento) discursiva:

(E.1.1)(E.1.3)(T) ...el fuerte embate tecnológico modificará pero no eliminará la utilización del libro. 
...el fuerte embate tecnológico modificará mas no eliminará la utilización del libro.

Ahora bien, ¿estructuras semánticas coincidentes en el esquema (proceso de planificación) aseguran ensayos individuales con argumentos coincidentes en fuerza (proceso de escritura)? Los tres estudiantes asumen los mismos tres argumentos aunque la justificación de cada uno revela la consideración de fuerzas diferentes (ver Tabla 3). Así, E1.1 los justifica en similares términos de extensión (A1: 27\%, A2: 25\%, A3: 21.9\%). Para E1.2, el argumento de mayor fuerza es el tercero (44.4\%), seguido del segundo (11.6\%) y del primero $(6 \%)$; un quinto argumento (A5), aportado únicamente por ella en el desarrollo de su texto (aunque no en el esquema), obtiene el segundo lugar de fuerzas (28.2\%); de ello se desprendería que fue una preocupación personal no discutida o, en cuya importancia, al menos, no hubo acuerdo.

(E1.2)(A5) Internet incide negativamente sobre el uso de la lengua y, específicamente, sobre el proceso comprensivo del lector, debido al almacenamiento rápido de información en el menor espacio posible.

Tabla 3. Distribución porcentual de la fuerza de los argumentos por estudiante.

\begin{tabular}{|c|c|c|c|c|c|c|c|c|c|c|c|c|c|}
\hline \multirow[b]{3}{*}{ Estudiante } & \multirow[b]{3}{*}{$\mathrm{N}^{\circ}$ palabras } & \multicolumn{12}{|c|}{ ARGUMENTOS } \\
\hline & & \multicolumn{2}{|c|}{ A1 } & \multicolumn{2}{|c|}{ A2 } & \multicolumn{2}{|c|}{ A3 } & \multicolumn{2}{|c|}{ A4 } & \multicolumn{2}{|c|}{ A5 } & \multicolumn{2}{|c|}{ A6 } \\
\hline & & $f$ & $\%$ & $f$ & $\%$ & $f$ & $\%$ & $f$ & $\%$ & $f$ & $\%$ & $f$ & $\%$ \\
\hline E1.1 & 356 & 96 & 26.97 & 89 & 25.00 & 93 & 26.12 & 78 & 21.91 & & & & \\
\hline E1.2 & 482 & 29 & 6.02 & 56 & 11.62 & 214 & 44.40 & 47 & 9.75 & 136 & 28.22 & & \\
\hline E1.3 & 307 & 80 & 26.06 & 55 & 17.92 & 48 & 15.64 & 124 & 40.39 & & & & \\
\hline E2.1 & 359 & 158 & 44.01 & 0 & 0.00 & 201 & 55.99 & & & & & & \\
\hline E2.2 & 403 & 267 & 66.25 & & & 136 & 33.75 & & & & & & \\
\hline E2.3 & 310 & 127 & 40.97 & 48 & 15.48 & 135 & 43.55 & & & & & & \\
\hline E3.1 & 509 & 109 & 21.41 & 0 & 0.00 & 42 & 8.25 & 103 & 20.24 & 221 & 43.42 & 34 & 6.7 \\
\hline E3.2 & 378 & 0 & 0.00 & 111 & 29.37 & 75 & 19.84 & 71 & 18.78 & 62 & 16.40 & 59 & 15.6 \\
\hline E3.3 & 356 & 44 & 12.36 & 0 & 0.00 & 146 & 41.01 & 0 & 0.00 & 98 & 27.53 & 68 & 19.1 \\
\hline E4.1 & 558 & 111 & 19.89 & 195 & 34.95 & 52 & 9.32 & 57 & 10.22 & 143 & 25.63 & & \\
\hline E4.2 & 335 & 130 & 38.81 & 44 & 13.13 & 44 & 13.13 & 31 & 9.25 & & & 86 & 25.7 \\
\hline E4.3 & 408 & 114 & 27.94 & 149 & 36.52 & & & & & 145 & 35.54 & & \\
\hline
\end{tabular}


Para E1.3, el argumento de mayor fuerza es el cuarto (A4), argumento solo incluido por él en su esquema, no obstante desarrollado por todos en el ensayo; a él le otorga la mayor fuerza (40.4\%). Ello indica que fue uno de los puntos tocados en la discusión, que llegaron al acuerdo de no incluirlo o lo vieron incluido en otro. Su proceso de justificación distribuye las fuerzas de los argumentos restantes: A1: 26.1\%; A2: 17.9\%; y A3: $15.6 \%$.

(E1.3)(A4) La información contenida en los libros está apoyada por el prestigio de la editorial. En Internet ésta no tiene respaldo alguno.

Las variaciones estilísticas que ocurren en este primer grupo se dan en la forma de los enunciados oracionales y revelan la recurrencia de los sujetos a sus actividades metasemántica y metapragmática, pues se presentan algunas matizaciones de sentido al elegir unas palabras en lugar de otras, expresiones definidas en lugar pronombres (o viceversa) y una conjunción que aparentemente formaliza el discurso en lugar de otra, de manera consciente e intencional, pues al hacerlo están optando por construcciones diferentes de las de sus compañeros.

4.2. Coincidencia total del grupo o de individuos de un mismo grupo y divergencia de otros tanto en la macroproposición como en la forma del enunciado oracional: Grupos 2 y 3

\subsubsection{Grupo 2}

En este grupo, E2.1 y E2.3 utilizan el mismo enunciado como tesis; en cambio, E2.2 parece sentir la necesidad de crear un enunciado con un mayor carácter informativo:

(E2.1)(E2.3) Los libros como fuente de conocimiento permanecerán a lo largo del tiempo, ya que la tecnología solo está ampliando las formas en que éstos se presentan al incluir el formato digital.

(E2.2) Los libros como fuente de conocimiento permanecerán a lo largo del tiempo, ya que la tecnología solo introduce un nuevo formato (el digital) que se une al impreso; aunque el digital está tomando mayor importancia, no terminará desplazando al impreso.

Además de la enunciación de una idea, la mayor importancia que está adquiriendo el formato digital, E2.2 fija con mayor contundencia su posición crítica: el no desplazamiento del formato impreso por el digital, posición velada en la tesis de sus compañeros, quienes, aunque plantean la incorporación del digital como una 'ampliación' de formatos, no asumen explícitamente la no desaparición del impreso en el futuro, pues su afirmación únicamente está en relación con la permanencia del libro 'como fuente de conocimiento'. De tal manera que, en términos de la 
teoría de la relevancia y de su definición del carácter ostensivo-inferencial de la comunicación, el enunciado de E2.2 pareciera reflejar una doble intención: una intención informativa, la de 'informar a un interlocutor de algo', y una intención comunicativa, la de “informar a un interlocutor de una intención informativa propia” (Wilson \& Sperber, 2004: 244). Así, el estudiante informa a la profesora no solo de una idea (el digital como nuevo formato de texto); también lo hace de la posición crítica que asume (el formato digital no desplazará al impreso) y, además, de su competencia en la construcción de un texto argumentativo, objeto de evaluación.

Otro tanto ocurre en lo relativo al primero de los argumentos (A1):

La tecnología, de la mano de Internet, ha facilitado el acceso a la información eliminando muchas barreras que antes existían, por lo que fomenta la lectura.

Los libros en formato digital disponen de muchas herramientas que facilitan la búsqueda de información y el acceso a ésta, además son una forma mucho más práctica de almacenar información.

Si bien todos construyen el argumento en referencia al tópico 'acceso a la información', E2.1 y E2.3 establecen una relación causa-efecto entre tal acceso y el fomento de la lectura. En cambio, E2.2 elimina tal relación al obviar el efecto e incorporar otros dos tópicos: la facilidad de su búsqueda y la practicidad de su almacenamiento. E2.3 elabora un nuevo argumento (el cuarto) para adicionar la idea de E2.2 pero con un matiz semántico expresado a través del adverbio "solo", lo que aminora la importancia de la tecnología y, por tanto, asegura la permanencia del formato impreso en el tiempo, lo que le permite mantener coherentemente su posición crítica. E2.2 sigue generando un texto de gran contenido informativo y pareciera que E2.3 comienza a compartir su intención.

¿A qué se debe esa motivación, principalmente de E2.2, de crear un texto de tal relevancia informativa? Consideramos tres aspectos en este punto. El primero está vinculado con las habilidades verbales de estos estudiantes. E2.1 es una estudiante con una mayor habilidad discursiva que la de sus compañeros, por lo que su confianza en una competencia que estima alta pudiera conferirle la potestad de crear un texto, que es un esquema de ideas, informativo en lo mínimo requerido, cuyo desarrollo macroestructural ofrecerá posteriormente en el ensayo. No siente la necesidad de ser absolutamente explícita como sí, visiblemente, sienten E2.3 y, en mayor grado, E2.2.

En segundo lugar, la fuerza del argumento, percibida estratégicamente desde el momento de la planificación del ensayo, podría tener, también, una cierta incidencia (ver Tabla 3). Mientras E2.1 utiliza un 44\% de su argumentación para desarrollar el primer argumento, lo que lo ubica 
como segundo en fuerza, E2.2 usa el 63.3\%; en cambio, E2.3 los considera argumentos muy cercanos en fuerza a A3 si nos atenemos a la extensión de cada uno: $41 \%$ para el primero y $43.6 \%$ para el tercero.

Por último (y asumiendo que otras razones personales, de tipo estilístico, puedan influir), a esto puede agregársele la situación discursiva en la que este, como texto académico, se inscribe: un estudiante, cuyo propósito principal es demostrar un dominio cognitivo al profesor que lo evaluará; conocimientos tanto del tipo de texto que está creando (superestructura del esquema de ideas), como de la temática que desarrollará en el que posteriormente creará (ensayo) debido a que debe justificar suficientemente su posición crítica. De modo que el primero debe ser lo suficientemente informativo para que dé cuenta de lo que será el segundo.

El segundo de los argumentos, no incluido por E2.2 en el esquema, no fue desarrollado por E.2.1 en el cuerpo del ensayo y apenas considerado por E.2.3. De ello tendríamos que inferir que, a diferencia de sus compañeros, E2.2 puede evaluar metatextualmente su futuro ensayo en el esquema inicial de ideas. Existe un procesamiento metacognitivo que lo guía en la consideración de cuáles serán las ideas que, con seguridad, tendrá la capacidad de desarrollar. Llama la atención que E2.1, teniendo una mayor habilidad composicional que la de sus compañeros, no haya desarrollado este argumento en su texto a pesar de haberlo incluido en su esquema. Pudo haber entendido el esquema como texto flexible, objeto de cambio; o, quizás, sus niveles de control para atender selectivamente a los diferentes aspectos que demanda la tarea fueron menores que los de sus compañeros, por cuanto no creyó verse forzada a resolver problemas de diferente complejidad discursiva (oracional y supraoracional). Esto pudo derivar en un exceso de confianza en sus procesos automatizados y, consiguientemente, en la no inclusión de un argumento que había previsto desarrollar (el segundo). Tal como lo concluye Allal (2000), si bien un alto nivel en el manejo de las habilidades básicas del lenguaje escrito permite anticipar un extenso número de transformaciones posibles, desarrollar mayores mecanismos de control relativos a la organización textual y emplear las estrategias de transformación de mayor complejidad (sustitución y reordenamiento), las diferentes fases de la producción textual parecen incidir sobre las estrategias de regulación empleadas. Y, en este caso, la actividad metalingüística producida en la fase de textualización no parece necesariamente congruente con la desplegada en la fase de planificación.

Finalmente, existe acuerdo en el tercer argumento no obstante haberlo construido con matices semánticos diferenciadores. E2.1 y E2.3 coinciden en la mayor parte de la elaboración del enunciado: 
$(\mathrm{E} 2.1)(\mathrm{A} 3)$

A pesar de la existencia de libros electrónicos, la mayoría de las personas prefiere libros impresos porque leer una pantalla de computadora no es lo mismo que leer un libro.

(E2.3)(A3) A pesar de la existencia de los libros electrónicos la mayoría de las personas prefieren los libros impresos porque leer en una pantalla puede resultarles incómodo.

La diferencia estaría establecida en la mayor especificidad del enunciado de E.2.3, quien explicita la relación causa-efecto que E2.1 generaliza: no ser lo mismo = incomodidad. Ahora bien, si revisamos el ensayo de cada uno de estos sujetos, nos damos cuenta de que E2.1 considera un conjunto de razones por las que los medios de lectura establecen preferencias por uno $u$ otro: posibilidad de dividir los contenidos, de hacer anotaciones al margen, de que el lector establezca su propio ritmo, de detenerse o devolverse, además de su fácil transporte y su no requerimiento de energía eléctrica; de tal enumeración detallada así como del número de palabras que utilizó para su desarrollo se colige que lo entienda como el argumento de mayor fuerza. E2.3 solo considera una causa, la 'incomodidad', reflejada, más específicamente, en dos aspectos: la necesidad de un medio de energía y la incidencia de la lectura en pantalla sobre las enfermedades visuales.

E2.2 no cree necesario partir de la reiteración de la proposición 'existencia de libros electrónicos', pues fue explicitada en A1, al tiempo que enumera una serie de razones que explicarían la 'comodidad' que ofrece leer un libro en papel: contacto directo con el libro, ver la tinta, hacer anotaciones en el libro, escapar de un mundo lleno de pantallas brillantes y la no necesidad de fuentes de energía para su uso:

En la actualidad, a muchas personas les resulta más cómodo leer un libro en papel que leerlo en un monitor.

Se observa, además de diferencias relacionadas con la importancia del argumento, esto es, con su fuerza, y, en consecuencia, con su justificación, algunas diferencias léxicas: opta, en el esquema, por el término 'monitor' en lugar de 'pantalla', vocablo que, en cambio, utiliza en el ensayo y de 'en papel' en lugar de 'impreso'.

Un factor importante que nos muestran las elecciones de este grupo es la consideración de la relevancia informativa $\mathrm{y}$, por tanto, la actividad metapragmática que despliegan. Como ha podido apreciarse, E2.2 se desmarca continuamente de sus compañeros al tratar de crear no solo estructuras diferentes en cuanto a la forma del enunciado oracional, sino también en lo tocante al contenido proposicional; un indicador de la fidelidad de los sujetos a sus estilos discursivos y de la (meta)cognición que demuestran tener en relación con la naturaleza opcional/ 
no opcional de las decisiones lingüísticas, lo que analizaremos a continuación más detalladamente en la producción del grupo 3.

\subsubsection{Grupo 3}

La tesis es retóricamente idéntica en los tres estudiantes de este grupo:

(E3.1)(E3.2)(E3.3)(T) Con la continua inserción de la tecnología digital dentro de los diferentes ámbitos de nuestra vida diaria, los libros en su formato tradicional serán desplazados poco a poco por innovaciones más eficientes y económicas.

Los aspectos diferenciales que se producen están relacionados, básicamente, con la selección, la organización discursiva y la fuerza -dado sus procesos de justificación- de los argumentos desarrollados en el ensayo. Además, E3.1 diverge de E3.2 y E3.3 en la creación de un esquema que, al tener menos en cuenta su carácter informativo, tiende a una mayor brevedad expresiva. Así, los argumentos 1 y 5 son ejemplo de ello:

(E3.1)(A1) La necesidad de proteger a la naturaleza lleva a un constante ahorro de papel.

(E3.2)(E3.3) La búsqueda de proteger a la naturaleza ha despertado el interés de los investigadores por crear nuevas tecnologías que reduzcan la utilización del papel, como el e-book e Internet.

(E3.1)(A5) La creación del libro electrónico e-book reemplazará el formato tradicional debido a los beneficios que ofrece.

(E3.2)(E3.3) La creación del libro electrónico e-book reemplazará el formato tradicional debido a los beneficios que brinda: pantalla de iluminación, adecuar el tamaño de la letra según el deseo del usuario, ofrece al usuario diccionarios, capacidad de almacenamiento de 20 títulos, el e-book garantiza que ni editores o autores pierdan ganancias.

En la construcción de ambos argumentos salta a la vista la elección léxica; si bien entre 'ofrece' y 'brinda' la relación de significados es directa por lo que no parece haber grandes diferencias, sí las hay entre 'necesidad' y 'búsqueda': la búsqueda puede o no ser producto de la necesidad; sin embargo, en este caso se asume que lo es debido al explicativo proporcionado por E3.2 y E3.3: la 'necesidad' de reducir el uso del papel despierta el interés de los investigadores por la ‘búsqueda’ de nuevas tecnologías.

Asimismo, las elecciones pueden llegar más allá de la palabra y darse en el enunciado oracional. En ejemplos como el que sigue, E3.1 generaliza la información en un intento por construir un argumento en lugar de su justificación: 
En ciertas cátedras el uso de material impreso o libros, ha sido reemplazado por métodos electrónicos, como la contabilidad.

(E3.2)(E3.3)(A3) El SIl en Argentina ha anunciado el lanzamiento de la contabilidad electrónica en reemplazo de la manual como medida para facilitar el cumplimiento tributario.

En lo concerniente a la incidencia de cada argumento en la construcción del texto se observan variaciones incluso entre E3.2 y E3.3, quienes parten de un esquema idéntico. Así, para E3.1 los argumentos 2, 3 y 6 son irrelevantes $(0 \%, 8.3 \%$ y $6.7 \%$, respectivamente); su discurso se centra prácticamente en A5 (43.3\%): “La creación del libro electrónico ‘e-book’ reemplazará el formato tradicional debido a los beneficios que ofrece". Le siguen dos argumentos en relación, A1 (21.4\%) y A4 (20.2\%), con una relevancia media:

La necesidad de proteger a la naturaleza lleva a un constante ahorro de papel.

La publicación de libros en Internet es mucho más económica y de fácil adquisición que imprimirlos masivamente, implicando el gasto de papel en tinta.

En el caso de A1, tal relevancia no está en función de su importancia para la progresión discursiva del texto, sino más bien de la incapacidad de E3.1 para articularlo dentro del conjunto; dificultad que hace patente al indicar al inicio de su argumentación: 'Comencemos por algo que no tiene que ver enteramente con el ámbito tecnológico'.

Otro tanto ocurre con E3.2, quien privilegia A2 (29.4\%) con citas y ejemplos, trata prácticamente por igual los argumentos 3, 4, 5 y $6(19.8 \%, 18.8 \%, 16.4 \%$ y $15.6 \%$ respectivamente), y no incluye $\mathrm{A} 1$ en el desarrollo de su ensayo. E3.3 desarrolla ampliamente A3 (41\%), menos ampliamente A5 (27.5\%), A6 (19.1\%) y A1 (12.4\%), y no considera A2 ni A4.

(E3.2)(E3.3)(A1) La búsqueda de proteger a la naturaleza ha despertado el interés de los investigadores por crear nuevas tecnologías que reduzcan la utilización del papel, como el e-book e Internet

(E3.2)(E3.3)(A2) La utilización de material multimedia implica mayores beneficios en el proceso de aprendizaje en comparación con el modo estático y secuencial del material impreso tradicional.

(E3.2)(E3.3)(A3) El Sll en Argentina ha anunciado el lanzamiento de la contabilidad electrónica en reemplazo de la manual como medida para facilitar el cumplimiento tributario.

(E3.2)(E3.3)(A4) La publicación de libros en Internet se ha vuelto una forma más sencilla y 
económica para la adquisición de los mismos, con respecto de la impresión masiva en papel.

(E3.2)(E3.3)(A5) La creación del libro electrónico e-book reemplazará el formato tradicional debido a los beneficios que brinda: pantalla de iluminación, adecuar el tamaño de la letra según el deseo del usuario, ofrece al usuario diccionarios, capacidad de almacenamiento de 20 títulos, el e-book garantiza que ni editores o autores pierdan ganancias.

(E3.2)(E3.3)(A6) Con el paso del tiempo, los periódicos y publicaciones impresas serán progresivamente descontinuadas y nuevas formas más innovadoras tomarán su lugar (prensa on line).

De la interpretación de la distribución porcentual correspondiente a la extensión de la justificación de los argumentos, lo que hemos determinado como su fuerza, en los tres grupos analizados hasta ahora, resulta la aparición de tres tipos de proceder que ponen en evidencia la actividad metalingüística de cada individuo y, por lo tanto, el carácter autorregulado de su escritura: (a) algunos disponen, desde el proceso de elaboración del esquema, de la posibilidad de poder evaluar los argumentos y, en consecuencia, de la habilidad (metalingüística) para determinar cuáles de ellos desarrollarán y en qué medida, lo que hace que los obvien o los escriban atendiendo a las sugerencias del grupo o a sus propias consideraciones, o que incorporen nuevos argumentos (propios) que hayan sido o no discutidos en grupo; (b) otros intentan ser fieles a los esquemas, por lo que tocan, aunque sea insuficientemente, como aseveración no justificada, el argumento escrito. Esta parece ser la estrategia discursiva con la que intentan mantener la propiedad de intertextualidad entre el esquema y el ensayo; y (c) los últimos, los menos, aunque saben que parten de un esquema grupal, no desarrollan en el texto del ensayo breve argumentos que no consideran relevantes o que les son difíciles de articular en el conjunto, debido, entre otras razones, a la progresión discursiva que ha tomado el texto en su realización escrita. Así, en el conocimiento de que construyen un texto que diverge del previsto en el esquema, no toman acciones que intenten hacer congruente la relación entre ambos.

\subsection{Coincidencia parcial entre las macroproposiciones y las formas de los enunciados ora- cionales entre algunos de los miembros del grupo o no coincidencia: Grupo 4}

Entre las estructuras semánticas y oracionales de las tesis de los tres estudiantes de este grupo existen claras discrepancias. Ya no solo se trata de elaborar estructuras con un mismo centro temático pero que divergen en tanto enunciado oracional. A diferencia de la tesis de E4.1, las de E4.2 y E4.3 aluden al problema planteado, la sustitución del libro en formato físico por el libro digital: 
El uso de nuevas tecnologías está cambiando nuestra visión del mundo, ampliando nuestro conocimiento e influyendo en la manera de cómo obtenemos este debido a la mayor accesibilidad a la información por parte del Internet. Internet, junto con otras nuevas tecnologías, cambiarán el modo en que accedemos a la información y leemos sustituyendo los medios físicos.

La tecnología, recalcando Internet, está afectando el modo de leer y escribir debido a la transición del uso de la información del ámbito físico al digital.

Los tres refieren dos conceptos en correspondencia, tecnología-Internet, pero su utilización en el texto adquiere funciones diferentes. La idea de E4.1 se construye a partir del 'uso de nuevas tecnologías', uso que incide sobre el cambio de 'nuestra visión del mundo' debido a dos razones: (a) ampliación de 'nuestro conocimiento', y (b) manera de obtención del conocimiento (accesibilidad a través de Internet). Así, Internet se presenta como un factor que influye solo sobre una de las causas, la segunda. De manera semejante, E4.3 entiende como primigenio el concepto de tecnología, donde incluye el de Internet y su influencia sobre 'el modo de leer y escribir'. E4.2 destaca la noción de Internet, aunque la considera dentro del conjunto de las 'nuevas tecnologías'.

Los textos son claras hechuras de las tesis que los sujetos plantearon y de los argumentos que previeron y que consideraron más fuertemente. E4.1 incluye en su tesis el argumento principal ('mayor accesibilidad a la información por parte de Internet'), lo que indica la fuerza de un argumento que se articula, desde el inicio, a la tesis (A2: 35\%; A1: 19.9\%):

(E4.1)(A1) La comodidad de la persona al momento de consultar un libro; es más práctico tener su contenido en un formato digital, con lo cual se sinterizaría el tamaño del libro.

(E4.1)(A2) Se obtiene más beneficios al poseer una mayor cantidad de libros (información) en un menor espacio, con lo cual se mejora la comodidad y la rapidez de la consulta.

E4.2 se refiere tanto al modo en que se accede a la información como al proceso de lectura ('cambiarán el modo en que accedemos a la información y leemos'); así, los dos argumentos relativos a estas ideas adquieren las mayores fuerzas (A1: 38.8; A6: 25.7\%):

(E4.2)(A1) La comodidad de la persona al momento de consultar un libro: es más práctico tener su contenido en un aparato (como una palm), se puede tener la información de muchos libros en un solo instrumento. 
El Internet aumenta la comodidad del usuario al encontrar la información, pero no al leerla. El cambio de los libros por las nuevas tecnologías puede tardar mucho; va a requerir de un largo proceso de adaptación de las personas y de las tecnologías.

No obstante, hay que indicar que el primero de los argumentos se vio claramente reforzado con A2 (13.1\%):

El acceso a la información se agiliza al tener que buscar en una base de datos. Una computadora puede tener la información de una biblioteca y su consulta es instantánea. Actualmente hay más facilidad para conseguir información por Internet que por los libros porque se distribuye instantáneamente.

E4.1 y E4.2 incluyen otro argumento de menor fuerza (A3; E4.1: 9.3\%; E4.2: 13.1\%) también relacionado con la información, aunque esta vez en lo referente a almacenamiento y duración:

Se posee una mayor durabilidad del material y la información si se traslada a un formato digital.

(E4.2)(A3) En una computadora la información puede durar más y se puede regar fácilmente para garantizar su conservación, mientras que un libro tiene que resistir problemas físicos.

E4.3 opta por la consideración de únicamente tres argumentos en clara correspondencia con una tesis donde privilegia la relación causa-efecto de la transición del formato físico al digital sobre el modo de leer y escribir y, específicamente, en lo que a propiedad y corrección en el uso de la lengua se refiere (A1: 27.9\%; A2: 36.5\%; A5: 35.5\%); preocupación compartida con E.4.1 (A5: $25.6 \%)$.

(E4.3)(A1) Los libros como fuente de información están dejando de ser imprescindibles; los están desplazando los buscadores que con pocas palabras 'clave' proveen toda la información que se quiera sin necesidad de buscarla en varios libros distintos.

(E4.3)(A2) La comodidad y practicidad que ofrece la tecnología lleva consigo la dejadez por la investigación y el cambio del concepto de 'búsqueda activa del conocimiento'.

(E4.3)(A5) La riqueza y variedad del lenguaje no se pone de manifiesto digitalmente del modo en que lo hace físicamente: cualquier persona tiene acceso a Internet para publicar escritos, que bien pueden estar mal elaborados, mientras que la información física tiene más garantía de una correcta estructuración. 
El uso de Internet tiende a influir en la manera de pensar del individuo, debido a la informalidad presente en la forma como se expresa su contenido.

Finalmente, E4.1 y E4.2 incluyen en su esquema otro argumento apenas justificado: A4 (10.2\% y $9.3 \%$, respectivamente).

Las escuelas están fomentando al estudiante el uso de las nuevas tecnologías en la búsqueda activa del conocimiento, prescindiendo cada vez más de los libros.

Entre los estudiantes de este grupo se mantiene el tópico pero con distintos sentidos locales, lo que hace que no se elaboren discursos diferentes. Las elecciones afectan la macroestructura del esquema debido a que son congruentes con la perspectiva de la que parten los escritores; habiendo variaciones en el sentido, es lógico que se produzcan enunciados oracionales diferentes también, pues ellos deben responder a las estructuras semánticas que intentan construir.

\section{CONCLUSIONES}

El carácter opcional de las decisiones lingüísticas fue concebido por los estudiantes con absoluta conciencia y, en consecuencia, tratado de manera controlada. Aunque se reunieron en grupos para planificar en forma conjunta un esquema de ideas, hicieron uso de la potestad que les brinda la lengua de elegir (intencionalmente) un término en lugar de otro, una construcción en lugar de otra o una idea en lugar de otra. Así, las elecciones lingüísticas operaron en todos los niveles de realización del enunciado: en el nivel de la palabra, para indicar percepciones semánticas diferentes ('pantalla/monitor'; 'la mayor/gran parte'), para formalizar (aunque inadecuadamente) la expresión ('mas/pero') o al hacer uso de estrategias de cohesión ('Internet-en la red/en ella'); en el de la cláusula, para establecer diferentes relaciones textuales, proposicionales; y, en el de la oración, en caso de concebir tesis diferentes o argumentos diferentes (en contenido y fuerza) para el desarrollo de una misma tesis. Por lo tanto, los estilos propios, el contexto situacional, las intenciones discursivas y los conocimientos previos tuvieron incidencia sobre la composición de los esquemas y, especialmente, sobre la de los ensayos en cuya escritura pudieron adquirir mayor independencia y, con ello, definirse con mayor amplitud y comodidad.

Los estudiantes debieron planificar la escritura de un ensayo, uno de los formatos en que se 
puede presentar el texto argumentativo. La intención persuasiva que mueve a los escritores de este tipo de texto puede explicar el hecho de que no haya habido acuerdo no tan solo en los argumentos que consideraron pertinentes para apoyar sus opiniones, sino ni siquiera en su fuerza, su relevancia, en caso de que coincidieran en la inclusión de un mismo argumento. Ello pudo haber repercutido, también, en la mayor o menor contundencia con que los sujetos fijaron sus posiciones en relación con uno de los tópicos y, consecuentemente, en la construcción de enunciados de mayor o menor extensión, de mayor o menor explicitud.

El procesamiento estratégico con que los estudiantes operaron durante la escritura del ensayo se hizo visible básicamente en tres aspectos: (a) fueron capaces de asumir una posición crítica, personal, frente al problema y elaborar una lista de argumentos acorde con los fundamentos de tal posición. (b) En situación de coincidencia grupal en la posición crítica, pudieron construir tanto tesis como argumentos de manera consensuada, al tiempo que llevaron a su escritura sus particularidades cognitivas y pragmáticas. Y, (c) en el nivel macroestructural (metatextual), la mayor parte de ellos fue capaz de evaluar, durante la creación del esquema, la pertinencia de la inclusión de algunos argumentos. De allí que optaran, en ciertos casos, por no anotar argumentos seleccionados por su(s) compañero(s) o por añadir otros al esquema acordado por el grupo. Solo algunos dejaron en sus esquemas argumentos que no fueron tratados en sus ensayos.

De tal manera que se interrelacionaron factores de orden pragmático, textual y cognitivo; los sujetos intentaron organizar el texto no solo en función de un contenido (macroestructura) que habría de ser congruente con la superestructura argumentativa, sino también en razón de las motivaciones pragmáticas (la situación en la que se inscribe el texto, el carácter ostensivo de la comunicación, las intenciones que, por ende, mueven a la escritura del texto, la fuerza con la que el emisor percibe cada argumento y su estilo discursivo), y de sus habilidades lingüísticocognitivas.

En cuanto a las habilidades lingüístico-cognitivas, habría que tener en cuenta el desempeño de los estudiantes en los diferentes niveles de estructuración del texto. Si bien ha tendido a establecerse una correspondencia entre la complejidad de los niveles de representación mental y la de los niveles de la lengua, los resultados de esta investigación nos permiten afirmar que pueden existir excepciones en tal sentido. Ello explicaría que, en algunos, la competencia demostrada en la fase de planificación no fuera necesariamente congruente con la competencia exhibida en la fase de textualización, en virtud de que en la segunda debe intervenir en mayor medida el conocimiento metagramatical del individuo, o de que los sujetos pueden no soler establecer planes de texto; de manera que la textualización no ve guiada su progresión discursiva por un esquema previo. Esto nos lleva a pensar en la pertinencia de considerar, en la 
enseñanza de la escritura como un proceso, además de las diferentes fases que actúan retroalimentándose unas de otras, los variados niveles de actividad metalingüística (metapragmática, metatextual y metagramatical, especialmente) con los que pueda operar el sujeto. Las actividades didácticas, en consecuencia, deben atender las necesidades grupales, pero también las individuales, muchas de las cuales se dan en el nivel local del discurso (microestructural) donde las restricciones, tanto gramaticales (relaciones de concordancia, formas verbales y uso de conectores, entre otros) como pragmáticas (estilo de un texto académico, formal, que exige de su emisor, por ejemplo, la aplicación de estrategias de sustitución lexical) a las que se ven sujetas las elecciones actúan con gran fuerza, y podrían limitar la toma de decisiones del sujeto para hacer efectiva su facultad de elegir.

A ello ayudará la reescritura de ambos textos, esquema y ensayo, tanto en el nivel macroestructural como en el microestructural. En el primero, el sujeto podrá darse cuenta, frente a la evidencia, de cuáles fueron las ideas verdaderamente relevantes para él, de su articulación en la progresión discursiva y temática del texto y, por consiguiente, operar cambios en alguno de los dos textos o en ambos: registrar nuevos argumentos, descartar otros, refrasear aquellos que se mantengan, reforzar la justificación de los que parecieran un tanto débiles, así como discriminar entre argumentos y estrategias argumentativas para su justificación. En lo tocante al segundo, podrá revisar con énfasis los enunciados oracionales, su cohesión.

\section{REFERENCIAS BIBLIOGRÁFICAS}

Allal, L. (2000). Regulación metacognitiva de la escritura en el aula. En M. Milian \& A. Camps (Comps.), El papel de la actividad metalingüística en el aprendizaje de la escritura (pp. 187-214). Buenos Aires: Homo Sapiens.

Arnoux, E., Nogueira, S. \& Silvestri, A. (2006). Comprensión macroestructural y reformulación resuntiva de textos teóricos en estudiantes de institutos de formación de docentes primarios. Revista Signos, 39(60), 9-30.

Arnoux, E., Borsinger, A., Carlino, P., Di Stefano, M., Pereira. C. \& Silvestre, A. (2004). La intervención pedagógica en el proceso de escritura de tesis de posgrado [en línea]. Disponible en: http://www.escrituraylectura.com.ar/posgrado/articulos.htm

Beke, R. \& Bruno, E. (2005). Desempeño de docentes y de bachilleres no docentes en la elaboración de resúmenes. Revista de Pedagogía, 26(75), 9-30.

Bialystok, E. (2001). Bilingualism in development. Language, literacy \& cognition. New York: Cambridge University Press.

Camps, A. (2001). El aula como espacio de investigación y reflexión. Barcelona: Graó. 
Camps, A., Guash, O., Milian, M. \& Ribas, T. (2000). Actividad metalingüística: La relación entre escritura y aprendizaje de la escritura. En M. Milian \& A. Camps (Comps.), El papel de la actividad metalingüística en el aprendizaje de la escritura (pp. 135-162). Buenos Aires: Homo Sapiens.

Camps, A. \& Ribas, T. (Dir.). (1996). La evaluación del aprendizaje de la composición escrita en situación escolar. Madrid: MEC-CIDE.

Carlino, P. (2003). Alfabetización académica: Un cambio necesario, algunas alternativas posibles. Educere, 20, 409-420.

Carlino, P. (2004). El proceso de escritura académica: Cuatro dificultades de la enseñanza universitaria. Educere, 26, 321-327.

Castelló, M. (Coord). (2007). Escribir y comunicarse en contextos científicos y académicos. Conocimientos y estrategias. Barcelona: Graó.

Castelló, M. \& Monereo, C. (1996). Un estudio empírico sobre la enseñanza y el aprendizaje de estrategias para la composición escrita de textos argumentativos. Infancia y Aprendizaje, 74, 39-55.

Flower, L. \& Hayes, J. (1996). La Teoría de la Redacción como proceso cognitivo. En R. Rudell, M. Rapp Rudell \& H. Singer (Eds.), Los procesos de lectura y escritura. Textos en contexto (pp. 73-107). Buenos Aires: IRA. Lectura y Vida.

Fuenmayor, G., Flores, Y. \& Rincón, M. (2008). Construcción de la microestructura y macroestructura semántica en textos expositivos producidos por estudiantes de LUZ. Letras, 77, $189-219$.

Gombert, J. (1992). Metalinguistic development. London: Harvester Wheatsheaf.

Hayes, J. \& Flower, L. (1980). Identifying the organization of writing processes. En L. Gregg \& E. Steinberg. (Eds.), Cognitive processes in writing: an interdisciplinary approach (pp.3-30). Hillsdale, NJ: Erlbaum.

Karmiloff-Smith, A. (1994). Más allá de la modularidad. Madrid: Alianza Editorial.

Kintsch, W. \& van Dijk, T. (1978). Toward a model of text comprehension and production. Psychological Review, 85, 364-394.

Milian, M. (1996). Incidencia del contexto en el proceso de producción de textos escritos. Cultura y Educación, 2, 67-78.

Milian, M \& Camps, A. (Comps.). (2000). El papel de la actividad metalingüística en el aprendizaje de la escritura. Buenos Aires: Homo Sapiens.

Miras, M. (2000). La escritura reflexiva. Aprender a escribir y aprender acerca de lo que se escribe. Infancia y Aprendizaje, 89, 65-80.

Morles, A., Arteaga, M., Bustamante, S. \& García, M. (2002). Relación entre el desempeño en la lectura y la escritura de los estudiantes venezolanos. Revista de Pedagogía, 23(67), 225-246. 
Poblete, C. (2005). Producción de textos argumentativos y metacognición. Letras, 71, 63-88.

Riestra, D. (1999). Reenseñar la escritura a estudiantes universitarios. Infancia y Aprendizaje, $88,43-56$.

Scardamalia, M. \& Bereiter, C. (1992). Dos modelos explicativos de los procesos de composición escrita. Infancia y Aprendizaje, 58, 43-64.

Serrano, S. (2003). Estrategias para la construcción del resumen. Un estudio con Docentes en Servicio. Letras, 67, 74-112.

Sperber, D. \& Wilson, D. (1986). Relevance. Communication and cognition. London: Basil Blackwell.

Tapia, M., Burdiles, G. \& Arancibia, B. (2003). Aplicación de una pauta diseñada para evaluar informes académicos universitarios. Revista Signos, 36(54), 249-257.

van Dijk, T. (2000). El estudio del discurso. En T. van Dijk (Comp.). El discurso como estructura y proceso (pp. 21-65). Barcelona: Gedisa.

Velásquez, M. \& Alonzo, T. (2007). Desarrollo y transferencia de estrategias de producción escrita. Revista Signos, 40(63), 219-238.

Verschueren, J. (2002). Para entender la pragmática. Madrid: Gredos.

Villegas, C. (2001). Desarrollo de procesos cognoscitivos a través de estrategias de aprendizaje fundamentadas en la lingüística del texto. Letras, 62, 137-158.

Wilson, D. \& Sperber, D. (2004). La teoría de la relevancia. Revista de Investigación Lingüística, $1,237-288$.

\section{NOTAS}

1 ..."algunas elecciones son prácticamente automáticas en comparación con otras que pueden tener alta motivación" (Verschueren, 2002: 298).

2 "Definimos código como cualquier variante distinguible de una lengua que supone un conjunto sistemático de elecciones, unida a un área geográfica específica, una clase social, unas funciones asignadas o a un contexto de uso específico" (Verschueren, 2002: 199).

3 Bialystok considera la actividad metalingüística como la designación de un proceso que puede moverse dentro de diferentes dominios que van de la oralidad a la escritura, de los usos de mayor informalidad hasta los de mayor formalidad. Definición que interrelacionamos, a efectos de esta investigación, con una de las definiciones de estilo que hemos adoptado, la aportada por Verschueren (2002). Así, las situaciones de escritura y, además, de formalidad discursiva demandarían una mayor actividad metalingüística al exigir altos niveles de control asociados con procesamientos de mayor intencionalidad por cuanto el sujeto debe centrar su atención en resolver problemas lingüísticos que no resuelve usualmente.

4 En esta oportunidad hemos simplificado la determinación del criterio relativo a la fuerza del 
argumento pues solo tomamos en cuenta el número de palabras con las que fue desarrollado. Partimos del supuesto de que 'a mayor extensión de la justificación mayor importancia', ya fuera por el contenido proposicional que aporta en la configuración del argumento, ya fuera por el conocimiento previo de que dispone el sujeto. En una próxima investigación, pretendemos determinar, a partir de un estudio centrado en el ensayo en lugar de en el esquema, las estrategias de justificación utilizadas por los estudiantes y su relación con los factores que intervienen en la consideración de la fuerza de los argumentos, pero ese es otro estudio. 